\title{
Influence of the soil properties on the seismic response of structures
}

\author{
W. Bourouaiah ${ }^{1} \cdot$ S. Khalfallah ${ }^{1} \cdot$ S. Boudaa ${ }^{1}$
}

Received: 10 September 2018 / Accepted: 15 July 2019 / Published online: 24 July 2019

(c) The Author(s) 2019

\begin{abstract}
The objective of this study is to model the interaction between a concrete wall and a soil under seismic loading using the finite element method. The stiffness matrix of the soil is integrated to that of the structure to formulate the stiffness matrix of the entire system. To simulate the dynamic of soil-structure interaction, a numerical program was developed for this concern. So to resolve governed dynamic equations, the central difference method is used to compute displacement, velocity, and acceleration fields of soil, interface medium, and concrete wall nodes. The significance of soil foundation-structure interaction over fixed-base structure analysis showed that the integration of soil and foundation produces considerable changes in the seismic response. Obtained results using the soil-structure model and impedance functions are compared to those of fixed-base structure. The purpose is to calibrate the effects of soil properties and the soil-structure interaction on the seismic response of the structure and on the interaction medium.
\end{abstract}

Keywords Soil-structure interaction · Soil properties · Central difference method $\cdot$ Impedance functions $\cdot$ Concrete wall · Structure $\cdot$ Seismic response $\cdot$ Finite element method

\section{Introduction}

In earthquake engineering, the dynamic soil-structure interaction is a primordial task that has attracted a great interest of researchers and engineers. This phenomenon is taken into account to improve the seismic response of structures and evaluate their vulnerability (Sharma 2017). When a structure on soil is subjected to seismic loading, foundation oscillates depending on the supporting and surrounding soil, the foundation, and inertia of the superstructure. So the dynamic response of structures built on soft soil may significantly differ from the fixed-base structures (Kwon and Elnashai 2017). This fact is principally due to the dissipated energy of flexibly supported structures.

The literature offers the direct method, the substructure method or the hybrid method to model soil-structure interaction. In the direct method, the structure and the soil region around it are modeled together. So the geometry, the properties of the soil, and the behavior of the medium, and the structure could be considered in a unique step (Gullu and

S. Khalfallah

khalfallah_s25@yahoo.com

1 Ecole Nationale Polytechnique de Constantine, Ali Mendjeli, Algeria
Pala 2014). When the substructure method is employed, the soil supporting foundation is described by impedance functions (Chatzigogos et al. 2007; Mahmoudpour et al. 2011). At last, the hybrid method considers the macro-element concept of the soil-structure interaction (Pecker 2010; Lu et al. 2016). This approach combines the soil half-space, the foundation, and the interface between the soil and the structure (Figini et al. 2012).

Methods analyzing the soil-structure interactions are regrouped into two categories: (1) analytical methods and (2) numerical methods. There was a considerable lack in high power computing machine, analytical methods were popular and can only be used to solve simple problems. With the known innovation in computer science and numerical methods, many simulations are mostly used to study the soil-structure interaction. Thus, modeling of the interaction problems is elaborated based on the dimensional concept of problems under static or dynamic loading. Moreover, various computational methods such as the finite difference method (Dolicanin et al. 2010; Challamel et al. 2015), the finite element method (Zienkiewicz and Taylor 2013; Smith et al. 2013), and the boundary element method (Fedeliński 2004; Gernot et al. 2008) have been employed to analyze the interaction problems. 
On the other hand, procedures of structural design under seismic loading based on the performance objective have been developed over the last two decades (Ghobarah 2001). The procedure of performance-based earthquake engineering can quantify the probabilistic future seismic performance of buildings by a combination of structural capacity and seismic domain (Zarein and Krawinkler 2009). Really, the structural performance under seismic loading is neatly affected by the soil-structure interaction and its surrounding. In this case, a simplified approach is presented to study the effect of the soil-structure interaction on the non-linear seismic response of reinforced concrete structure (Mekki et al. 2014). Later, an approximate approach is formulated to analyze the soil-structure interaction and to evaluate the relative importance of its effects on structural performance (Mekki et al. 2016). In the same way, the assessment of the seismic performance considering the soil-structure interaction is studied to quantify the contribution of the soil Poisson' ratio, density of soil, shear wave velocity, soil dumping, and structure dumping on the lateral response of structure (Zoutat et al. 2016). Moreover Moghaddasi et al. (2012) have already defined the correlation between soil, structure, and interaction effects on the structural response where the effect of soil properties, structure characteristics, and their interaction on structural response have been analyzed.

This contribution is a prolongation of our developed investigation to analyze the effect of mechanical properties of soil on concrete wall responses under static loadings (Bourouaiah et al. 2017). In this way, the finite element model of soil-wall structure is integrated in the developed numerical program to study the seismic performance of structures considering the soil-structure interaction. The soil and the concrete wall were modeled using eight-node elements with two degrees of freedom for each node. The dimensional problem is justified by accuracy and satisfaction of results obtained by various studies using two-dimensional analysis for simple and regularly problems (Wood 2004).

\section{Modeling of the soil-structure interaction}

The resistance of ground to forces generate by different movements may transmit additional forces to the superstructure. This transmission of forces between the soil and the adjacent structures continues until the equilibrium of the soil-structure system is achieved. The energy transfer

Fig. 1 Soil-structure interaction modeling mechanism from the soil to the building during seismic loading is critical for design and conception of a structure. In this case, the behavior of the structure and the soil medium is greatly different, which can engender a relative response between them. Therefore, the approach to model rigorously the soil-structure interaction can be selected by providing accurate analytical or experimental results.

To understand the behavior of the soil-structure interaction, accurate modeling of the interface is recommended. Therefore, a rigorous technique of modeling and a constitutive behavior of the soil-structure medium have become a great concern. So, experimental studies have shown that a transition zone exists along the interface between the soil and the foundation fearing that for a small change of the thickness could produce large difference in obtained results (Hu and Pu 2004; Mayer and Gaul 2005) (Fig. 1a).

Various researches using the finite element method have been elaborated to investigate the soil-structure interaction problems. In this case, models can be regrouped in (1) modeling of the soil-structure interaction using node-to-node fulfilling compatibility conditions. This kind is quite often in practical applications (Langen and Vermeer 1991; Day and Potts 1994). These models are inspired from the relationship between relative displacements and stresses of common nodes (Fig. 1a). (2) In other cases, the behavior of the interface may be modeled by conventional finite element meshing conveying suitable mechanical properties of the contact media. They are used to predict the effect of the soil-structure interaction with interface elements and constitutive law. This approach is well established and largely used in finite element codes. In these analysis, the failure can occur in the nearest stress point for weak mechanical properties of elements (Viladkar et al. 1994; Skejic 2012; Barros et al. 2017; Sharma 2017) (Fig. 1b).

Finally, the zero-thickness interface elements or thin-layer elements are used to model the behavior of the discontinuity of joints in rock mechanics (Viladkar et al. 1994; Bouzid et al. 2004; Skejic 2012; Barros et al. 2017; Sharma 2017). These elements are used with respect to the finite element formulation, which can be triangular finite elements of 6-15 nodes or quadrilateral elements with 4,8 , and 9 nodes (Fig. 1c).

In this work, the modeling of the soil-structure interaction uses the direct method involving the soil and the concrete wall components in the same phase. Also, the nodeto-node contact elements for modeling the behavior of the

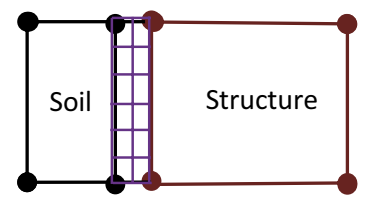

(b) Continuum elements

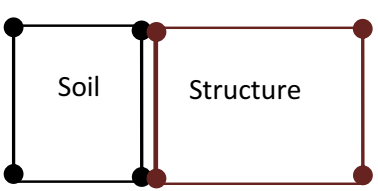

(c) Interface elements 
interface are employed for their suitability with that of the selected numerical method. Then the concrete wall is modeled by two-dimensional plane stress quadrilateral finite elements; even soil elements are modeled using plane strain elements.

\section{Numerical formulation}

The finite element method is a numerical powerful tool, largely used in the solid mechanic field. It is based on the approximation of the domain into various conventional finite elements. Thus, the finite element approach is an approximation of the domain $(D)$ involving the problem into nodal variables.

$\forall M(x, y) \in D:\left\{u_{n}^{*}(x, y)\right\}=[N(x, y)]\left\{u_{n}\right\}$,

where $\left\{u_{n}^{*}(x, y)\right\}$ is the approximate displacement vector, $[N(x, y)]$ the matrix of the interpolation functions, and $\left\{u_{n}\right\}$ is the nodal displacement vector of a finite element having $n$ node.

When a plane finite element with n nodes is used to simulate a problem, the shape function matrix can be written as:

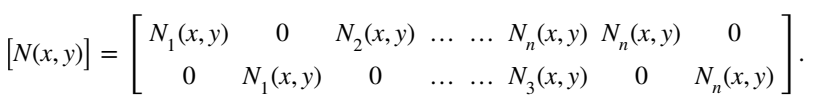

Corresponding to the natural system axis, the isoparametric shape function matrix (2) becomes

$[N(\xi, \eta)]=\left[\begin{array}{ccccccc}N_{1}(\xi, \eta) & 0 & N_{2}(\xi, \eta) & \ldots & \ldots & N_{n}(\xi, \eta) & 0 \\ 0 & N_{1}(\xi, \eta) & 0 & \ldots & \ldots & 0 & N_{n}(\xi, \eta)\end{array}\right]$

From the linear theory of elasticity, the deformation vector can be deduced as

$\{\varepsilon\}=[\partial]\left\{u^{*}(\xi, \eta)\right\}$.

The differential operator $[\partial]$ is $\left[\begin{array}{cc}\frac{\partial}{\partial \xi} & 0 \\ 0 & \frac{\partial}{\partial \eta} \\ \frac{\partial}{\partial \eta} & \frac{\partial}{\partial \xi}\end{array}\right]$.

Substituting Eq. (1) and considering the relation (3) into the Eq. (4), we obtain the differential interpolation function matrix which relies on strain and nodal displacement vectors.

$[B(\xi, \eta)]=\left[\begin{array}{cccccccc}\frac{\partial N_{1}(\xi, \eta)}{\partial \xi} & 0 & \frac{\partial N_{2}(\xi, \eta)}{\partial \xi} & 0 & \ldots & \ldots & \frac{\partial N_{n}(\xi, \eta)}{\partial \xi} & 0 \\ 0 & \frac{\partial N_{1}(\xi, \eta)}{\partial \eta} & 0 & \frac{\partial N_{2}(\xi, \eta)}{\partial \eta} & \ldots & \ldots 0 & \frac{\partial N_{n}(\xi, \eta)}{\partial \eta} \\ \frac{\partial N_{1}(\xi, \eta)}{\partial \eta} & \frac{\partial N_{1}(\xi, \eta)}{\partial \xi} & \frac{\partial N_{2}(\xi, \eta)}{\partial \eta} & \frac{\partial N_{2}(\xi, \eta)}{\partial \xi} & \ldots & \ldots & \frac{\partial N_{n}(\xi, \eta)}{\partial \eta} & \frac{\partial N_{n}(\xi, \eta)}{\partial \xi}\end{array}\right]$.
So, the stress vector integrating the constitutive material law can be deduced as:

$\{\sigma\}=[D]\{\varepsilon\}$.

$[D]$ is the elasticity matrix that can be written as:

For the plane stress state,

$[D]=\frac{2 G}{(1-v)}\left(\begin{array}{ccc}1 & v & 0 \\ v & 1 & 0 \\ 0 & 0 & \frac{1-v}{2}\end{array}\right)$

and for the plane strain state,

$[D]=\frac{2 G(1-v)}{(1-2 v)}\left(\begin{array}{ccc}1 & \frac{v}{1-v} & 0 \\ \frac{v}{1-v} & 1 & 0 \\ 0 & 0 & \frac{1-2 v}{2(1-v)}\end{array}\right)$,

where $G$ and $v$ are the shear modulus and Poisson ratio of the material used, respectively.

Therefore, the stiffness $\left[K_{\mathrm{e}}\right]$ and mass $\left[M_{\mathrm{e}}\right]$ matrices for each element and the load vector in natural coordinates are expressed by the following expressions:

$\left[K_{\mathrm{e}}\right]=t \int_{-1}^{+1} \int_{-1}^{+1}[B(\xi, \eta)]^{T}[D][B(\xi, \eta)]|J| \mathrm{d} \xi \mathrm{d} \eta$

$\left[M_{\mathrm{e}}\right]=\rho \int_{-1}^{+1} \int_{-1}^{+1}[N(\xi, \eta)]^{T}[N(\xi, \eta)]|J| \mathrm{d} \xi \mathrm{d} \eta$

$\left\{F_{\mathrm{e}}\right\}=\int_{-1}^{+1} \int_{-1}^{+1}[N(\xi, \eta)]^{T}\{f\}|J| \mathrm{d} \xi \mathrm{d} \eta$

$|J|$ is the determinant of the Jacobian matrix, $t$ and $\rho$ is the thickness of the element and the density of the used material, respectively.

Introducing Eq. (3) into the relationship (8b) for a quadrilateral finite element, the consistent stiffness matrix of a finite element can be evaluated as:

$\left[M_{\mathrm{e}}\right]=\frac{t \rho \Omega}{36}\left[\begin{array}{rrrrrrrr}4 & 0 & 2 & 0 & 1 & 0 & 2 & 0 \\ & 4 & 0 & 2 & 0 & 1 & 0 & 2 \\ & 4 & 0 & 2 & 0 & 1 & 0 \\ & & & 4 & 0 & 2 & 0 & 1 \\ & & & & 4 & 0 & 2 & 2 \\ & & & & & 4 & 0 & 2 \\ & & & & & & 4 & 0 \\ \text { Sym } & & & & & & & 4\end{array}\right]$. 
In the same way, the formulation of the stiffness matrix can be developed by substituting Eqs. (5) and (7a, 7b) into Eq. (8a). To analyze planed problems, two cases can be distinguished as:

Case 1 Plane stress cases viscous damping matrix, $\{u\}$ the relative nodal displacement vector, and $\left\{\ddot{u}_{\mathrm{g}}\right\}$ is the acceleration vector applied at the base of the wall structure. The solution of the dynamic response of the structural system (11) can be obtained by the

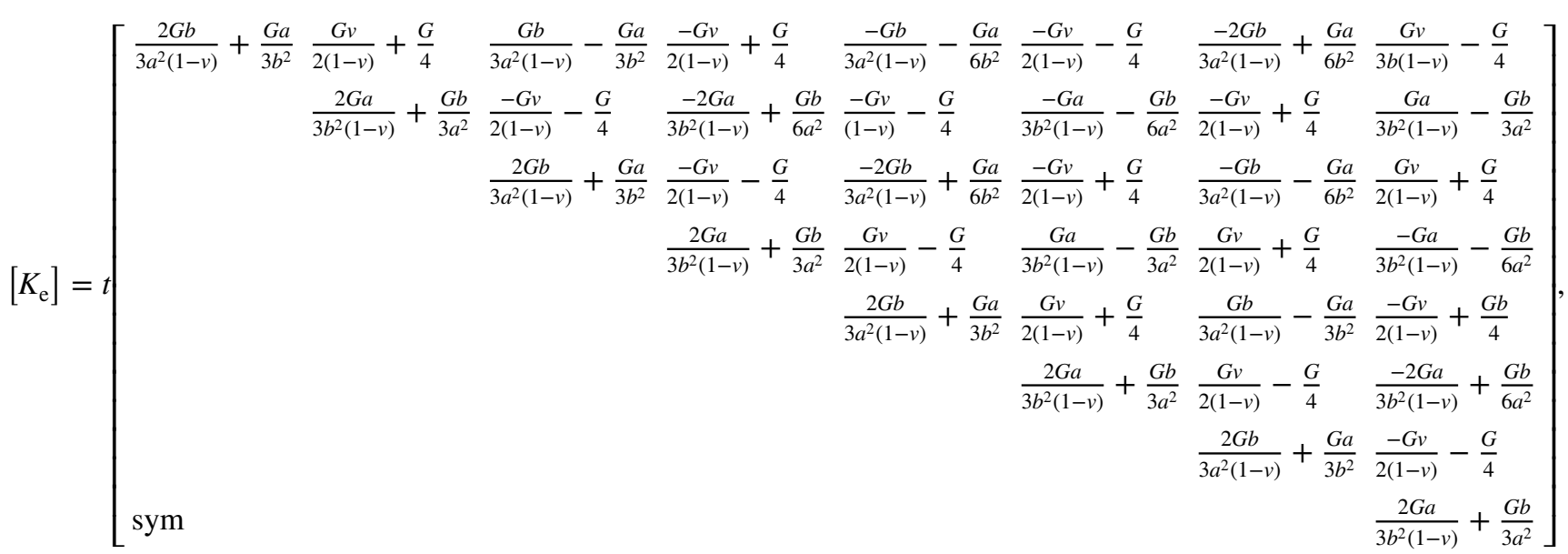

Case 2 Plane strain cases

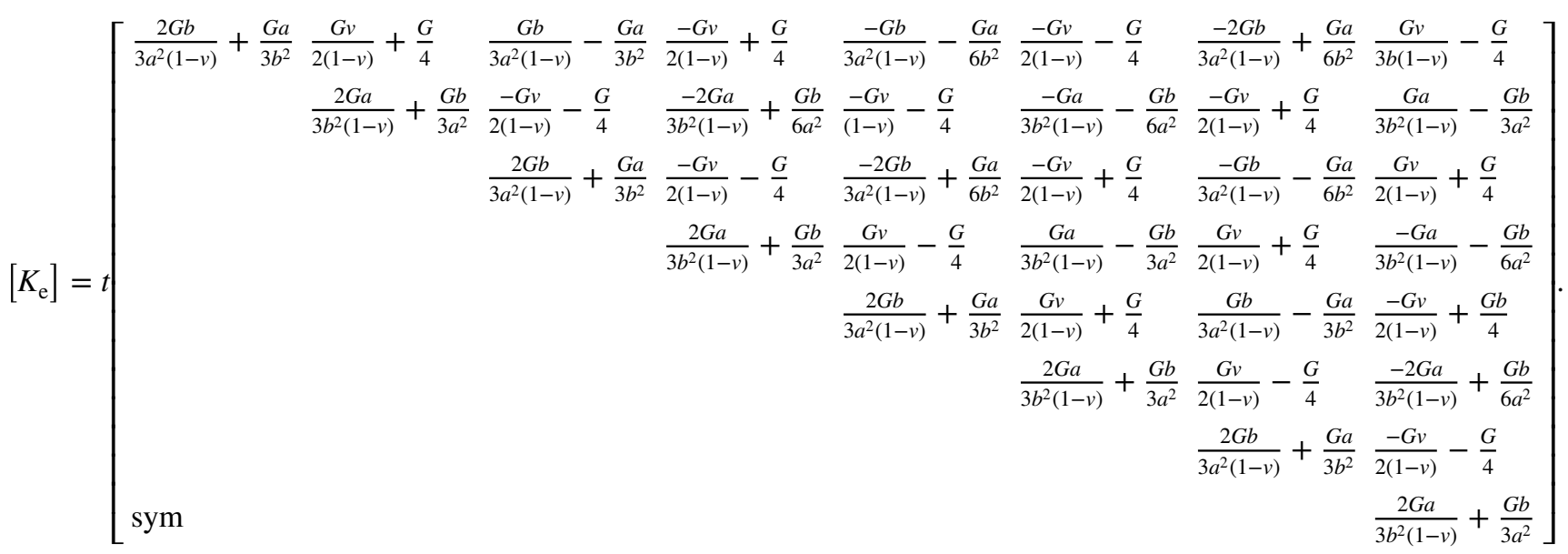

\section{Numerical dynamic analysis}

The dynamic or seismic response of the soil and concrete wall can be estimated by using the numerical solution in the time domain.

$[M]\{\ddot{u}(t)\}+[C]\{\dot{u}(t)\}+[K]\{u(t)\}=-[M]\left\{\ddot{u}_{\mathrm{g}}\right\}$,

where $[M]$ and $[K]$ are the mass and stiffness matrices of the entire structure defined by Eq. (9) and (10a, 10b), $[C]$ the direct numerical integration approach. Most methods use equal time intervals $\Delta t, 2 \Delta t, \ldots, n \Delta t$, and many numerical techniques have previously been presented. All approaches can be classified as either explicit or implicit integration methods, and implicit approaches attempt to satisfy the differential equation at time $t$ considering the solution at time $t-\Delta t$. Thus, these methods need the solution of a set of linear equations at each time step.

The numerical solution can be established using the central difference method to compute the solution of the dynamic 


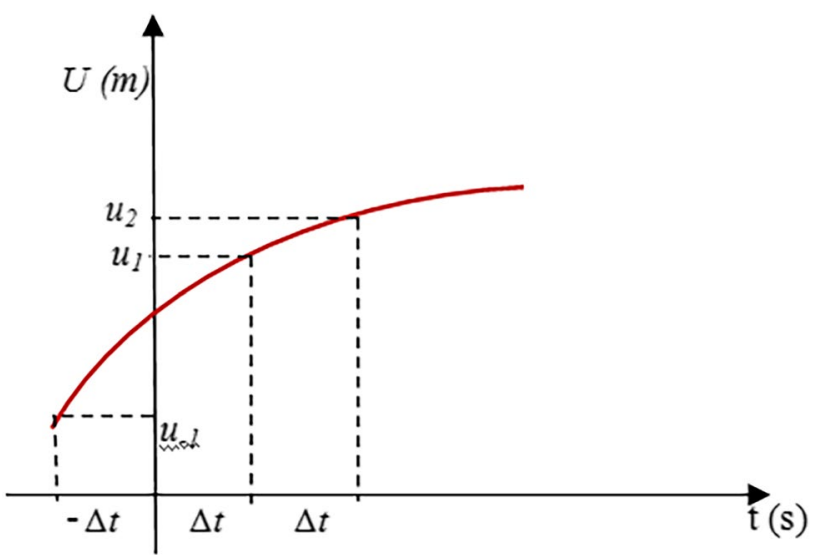

Fig. 2 Displacement-time relationship used in the central difference method

response of the structural system for its stability for real dynamic time and its implementation in the developed numerical program (Wu et al. 2009; Grobeholz et al. 2015).

\section{Central difference method}

For un-damped systems, the equation of motion (11) resulting from the finite element discretization is as follows:

$M \ddot{u}(t)=F_{\text {ext }}\left(\dot{u}_{n}(t), u_{n}(t)\right)-F_{\text {int }}\left(\dot{u}_{n}(t), u_{n}(t)\right)$.

The central finite difference method (Fig. 2) can be introduced in the Newmark scheme using $\gamma=1 / 2$ and $\beta=0$.

At the time $(n+1)$, velocity, and displacement expressions can be expressed by

$\dot{u}_{n+1}=\dot{u}_{n}+\frac{\Delta t}{2}\left(\ddot{u}_{n}+\ddot{u}_{n+1}\right)$,

$u_{n+1}=u_{n}+\Delta t \dot{u}_{n}+\frac{\Delta t^{2}}{2} \ddot{u}_{n}$.

The central difference method is based on the approximation on the velocity and displacement fields. Thus, the expressions of velocity are obtained as:

$\dot{u}_{n}-\frac{1}{2}=\frac{u_{n+1}-u_{n}}{\Delta t}$,

$\dot{u}_{n}+\frac{1}{2}=\frac{u_{n+1}-u_{n}}{\Delta t}$

Also, the acceleration expression at the time can be expressed using the displacement expression

$\ddot{u}_{n}=\frac{u_{n+1}-2 u_{n}+u_{n-1}}{\Delta t^{2}}$.

The expression of the velocity can be deduced using relations (13a, 13b) and (15)

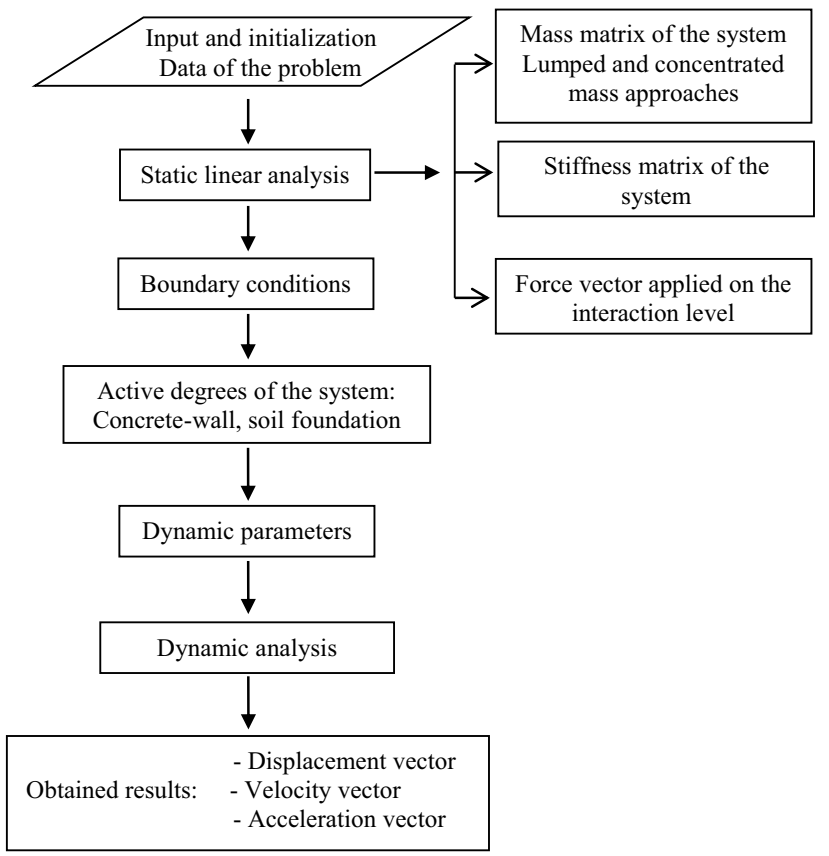

Fig. 3 Flowchart of the numerical program

$\dot{u}_{n}=\frac{u_{n+1}-u_{n-1}}{2 \Delta t}$.

The initial conditions can be considered as $u_{0}=0$ and $\dot{u}_{0}=0$ which lead to

$\ddot{u}_{0}=M^{-1}\left(K u_{0}+F\right)$.

The internal force vector can be computed by

$F_{0}=F-K\left(u_{0}+\Delta t u_{0}+\frac{1}{2}(1-\beta) \Delta t^{2} \ddot{u}_{0}\right)$,

$F_{n}=F-K\left(u_{n}+\Delta t \dot{u}_{n}+0.5(1-\beta) \Delta t^{2} \ddot{u}_{n}\right)$.

The corresponding acceleration and velocity are, respectively,

$\ddot{u}_{n+1}=\frac{F_{n}(i)}{K(i, i)}$,

$\dot{u}_{n+1}=\dot{u}_{n}+\Delta t(1-\gamma) \ddot{u}_{n}+\gamma \Delta t \ddot{u}_{n+1}$.

So, the expression of displacement at time $n+1$ is

$u_{n+1}=u_{n}+\Delta t u_{n}+0.5 \Delta t^{2} \ddot{u}_{n}$.

\section{Technique of the resolution}

To obtain results, a numerical program has been conceived for this concern. The flowchart of the program is based on the main parts constituting finite element numerical codes. 


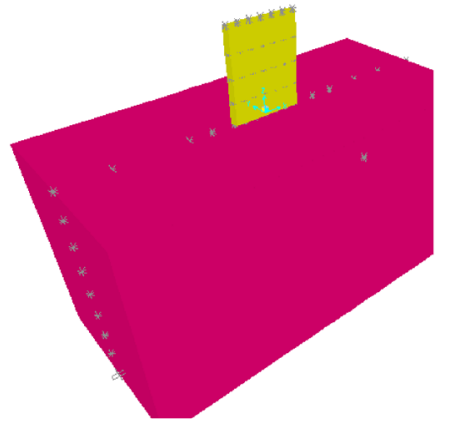

(a) Structure and soil foundation

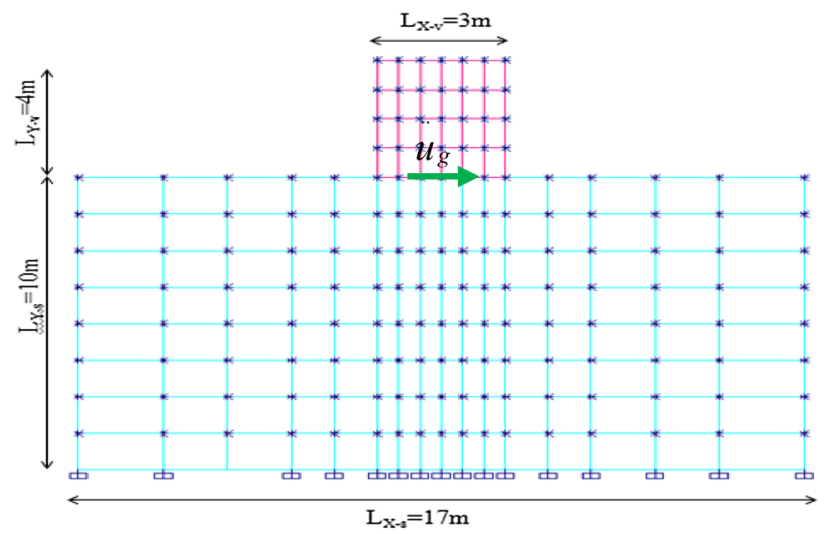

(b) Meshing of the structure and the soil

Fig. 4 Meshing of the concrete wall and the soil foundation

Table 1 Geometrical and mechanical properties of soil and concrete wall structure

\begin{tabular}{lllll}
\hline Material properties & $\begin{array}{l}\text { Geometrical } \\
\text { dimensions } \\
L_{x} \times L_{y} \times t(\mathrm{~m})\end{array}$ & $\begin{array}{l}\text { Shear modulus }(G) \\
\left(\mathrm{kN} / \mathrm{m}^{2}\right)\end{array}$ & $\begin{array}{l}\text { Poisson's ratio } \\
\nu\end{array}$ & $\begin{array}{l}\text { Density, } \rho \\
\left(\mathrm{kN} / \mathrm{m}^{3}\right)\end{array}$ \\
\hline Soil (S1) & $17 \times 10 \times 8$ & $19.2310^{5}$ & 0.45 & 15 \\
Soil (S2) & $17 \times 10 \times 8$ & $35.7110^{5}$ & 0.40 & 18 \\
Soil (S3) & $17 \times 10 \times 8$ & $74.0710^{5}$ & 0.35 & 20 \\
Soil (S4) & $17 \times 10 \times 8$ & $107.1410^{5}$ & 0.30 & 22 \\
Concrete & $3 \times 4 \times 0.3$ & $12510^{5}$ & 0.20 & 23 \\
\hline
\end{tabular}

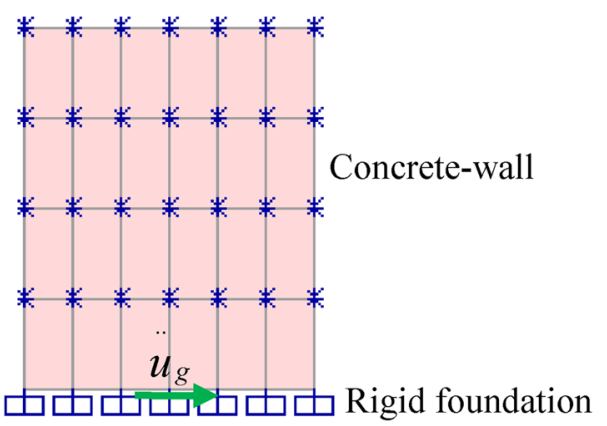

Fig. 5 Concrete wall with fixed base

Even linear analysis algorithm of the resolution is composed of four steps: (1) the presentation of the problem data, (2) the elaboration of the static linear analysis, (3) the dynamic elastic analysis, and (4) the presentation of dynamic results. All steps can be regrouped in the following flowchart (Fig. 3).

\section{Description of studied cases and modeling}

In this study, the dimensions of the concrete wall are $3 \times 4 \times 0.3 \mathrm{~m}$ and those of the soil are $17 \times 10 \times 8 \mathrm{~m}$ (Fig. 4a). The finite element meshes of the concrete wall and the soil are shown in Fig. 4b. In addition, the mechanical properties for soils used in this analysis are regrouped in Table 1. The soils are classified into four categories based on the shear modulus sweeping from the soft soil to the stiff one. Finally, the external loading is a seismic acceleration applied at the interface level between the foundation and the structure (Fig. 4b).

In this model, the soil medium and the concrete wall were discretized into quadrilateral finite elements (Fig. 4b). To satisfy boundary conditions, the located soil nodes at lateral sides were fixed against horizontal displacement while nodes of longitudinal side boundary were restrained in vertical and longitudinal displacements.

The concrete wall with fixed base is also studied to quantify the contribution and the effect of the soil on the concrete wall and the soil-structure interaction responses (Fig. 5).

\section{Results and discussions}

\section{Effect of soil properties on the interface media}

The aim of this study is to explain the effect of soil properties on the seismic response of the interface medium. Obtained results of this simulation using the central difference 
(a)

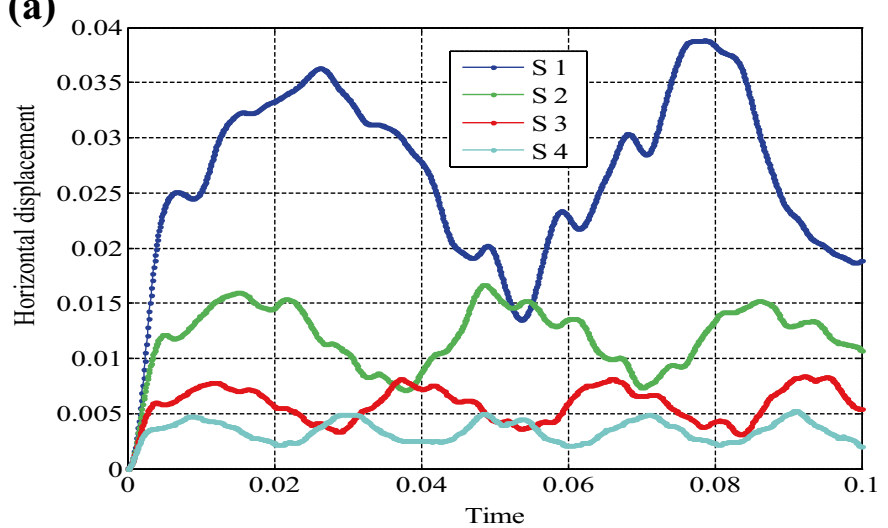

(b)

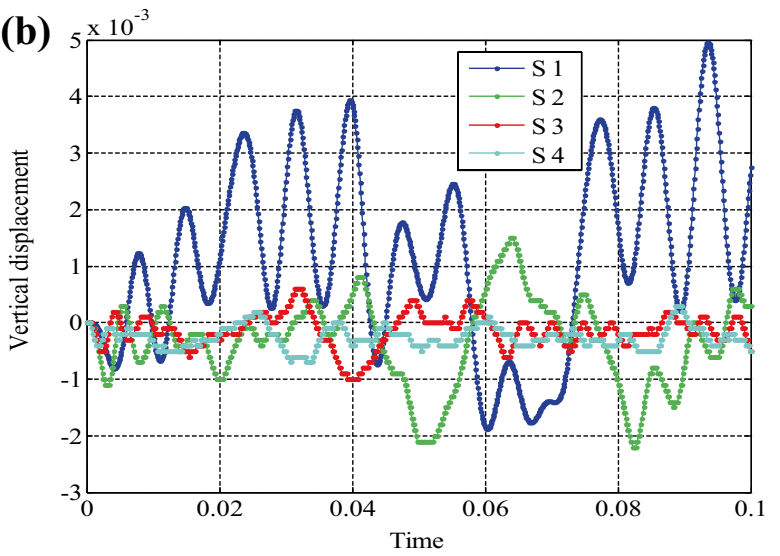

Fig. 6 Interface node displacements: a horizontal displacement $\mathbf{b}$ vertical displacement

(a)

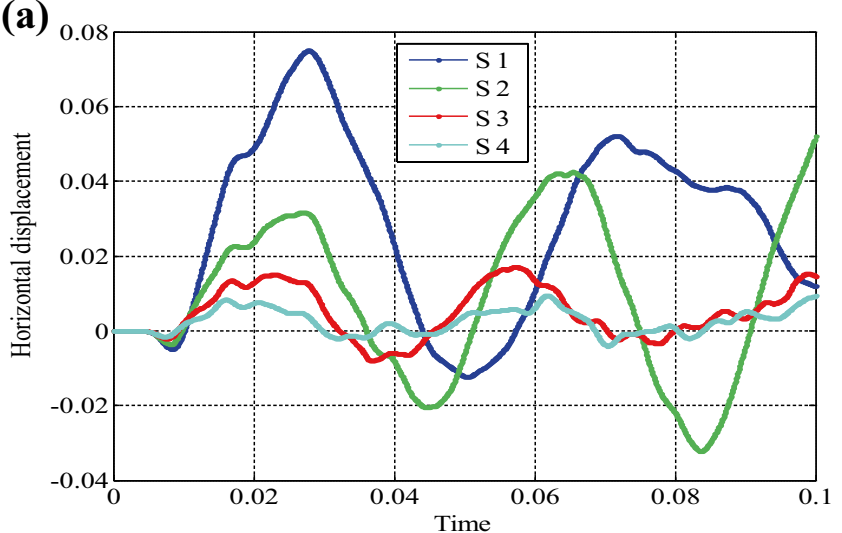

(b)

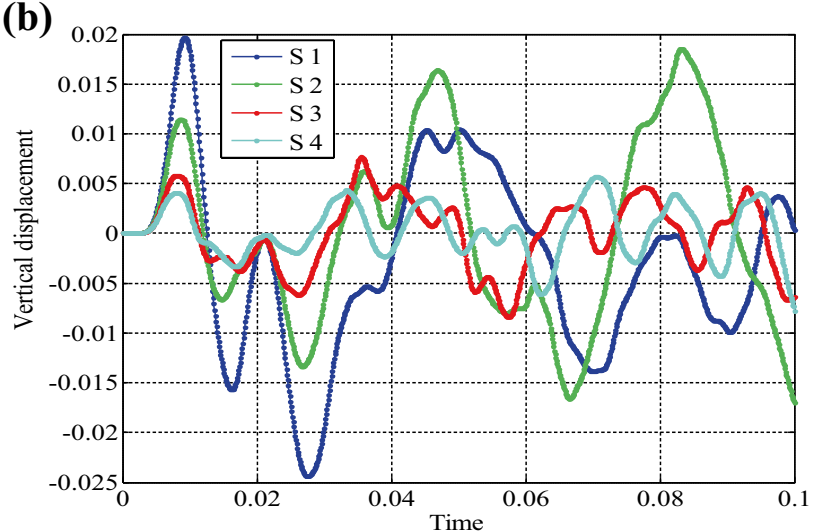

Fig. 7 Concrete wall node displacements: a horizontal displacement b vertical displacement

Table 2 Effect of soil properties on horizontal displacements

\begin{tabular}{lllll}
\hline Soil used & $\begin{array}{l}\text { Very soft soil } \\
(\mathrm{S} 1)\end{array}$ & Soft soil (S2) & Firm soil (S3) & Stiff soil (S4) \\
\hline Peak displacement (m) & 0.078 & 0.041 & 0.018 & 0.01 \\
Displacement ratio & 1.00 & 1.90 & 4.34 & 7.80 \\
\hline
\end{tabular}

approach are shown in Fig. 6. As a result, the horizontal and vertical displacements of interface nodes between soil and structure are depicted.

Obtained results show that soil proprieties have a great influence on the behavior of the interface medium and they are proportional to the soil nature. The peaks of oscillations are very important for soft soil and decrease as well as the soil becomes very stiff (Fig. 6). The seismic force is applied in the horizontal direction, so horizontal displacements are very significant compared to the vertical ones (Fig. 6a, b). Moreover, when the soil varies from S2 to S4, the difference between vertical displacements is not remarkable (Fig. 6b), but horizontal displacements are underlined (Fig. 6a).

\section{Effect of soil properties on the concrete wall}

In the same context, the properties of soil influence the seismic responses of concrete wall nodes. The concrete wall node displacements are very important in the horizontal direction (Fig. 7a) compared to those in the vertical direction (Fig. 7b). In addition, the horizontal and vertical displacements of the concrete wall are very important for S1 and decrease as the soil properties become more important 


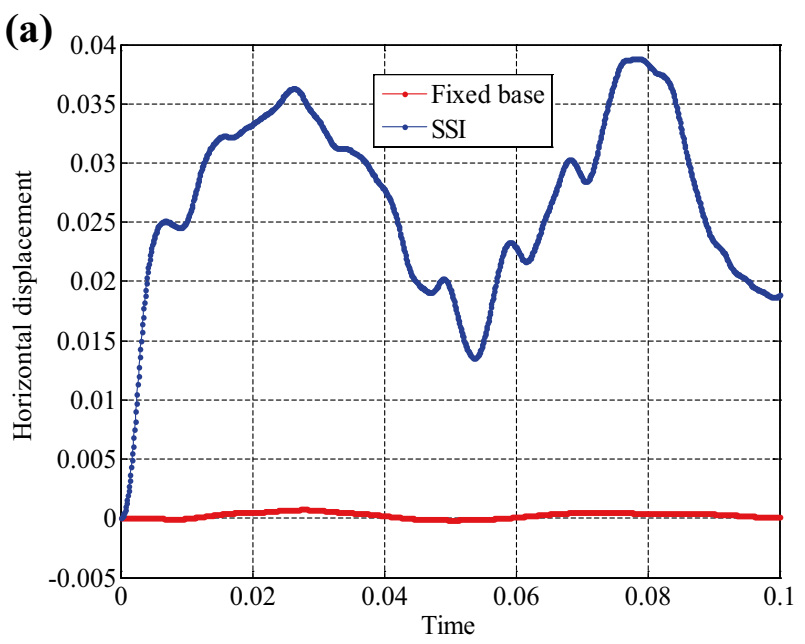

Fig. 8 Horizontal displacements: a interface nodes, b concrete wall nodes

Table 3 Soil stiffness under rectangular foundation

\begin{tabular}{lllll}
\hline Nature of soil & Very soft soil & Soft soil & Firm soil & Stiff soil \\
\hline Density, $\rho\left(\mathrm{KN} / \mathrm{m}^{3}\right)$ & 15 & 18 & 20 & 22 \\
Poisson ratio, $v$ & 0.45 & 0.40 & 0.35 & 0.30 \\
$a / b=2$ & & & & \\
$K_{h}\left(10^{6} \mathrm{kN} / \mathrm{m}\right)$ & 1.38 & 5.36 & 22.12 & 97.33 \\
$K_{v}\left(10^{6} \mathrm{kN} / \mathrm{m}\right)$ & 2.03 & 7.46 & 28.44 & 125.16 \\
\hline
\end{tabular}

(Fig. 7). Table 2 illustrates the influence ratio of the soil properties on the horizontal behavior of the concrete wall.

It is emphasized here that the design of the concrete wall with respect to the horizontal behavior must be taken into consideration.

\section{Influence of the soil-structure interaction}

Figure 8 quantifies the horizontal contribution of the soil-structure interaction on the seismic response of the interface medium and the wall structure. The soil-structure interaction model has an influence on the wall structure compared to the interface medium. They are about $3.88 \%$ and $7.49 \%$ for interface medium and concrete wall, respectively.

\section{Modeling of the soil foundation}

The developed program can be also used to evaluate the soil-structure interaction and concrete wall responses substituting the soil foundation per equivalent stiffness parameters (Table 3). In this case, translational springs have been integrated according to the plane directions such as $K_{h}$ and $K_{v}$ (Fig. 9), which are used to substitute
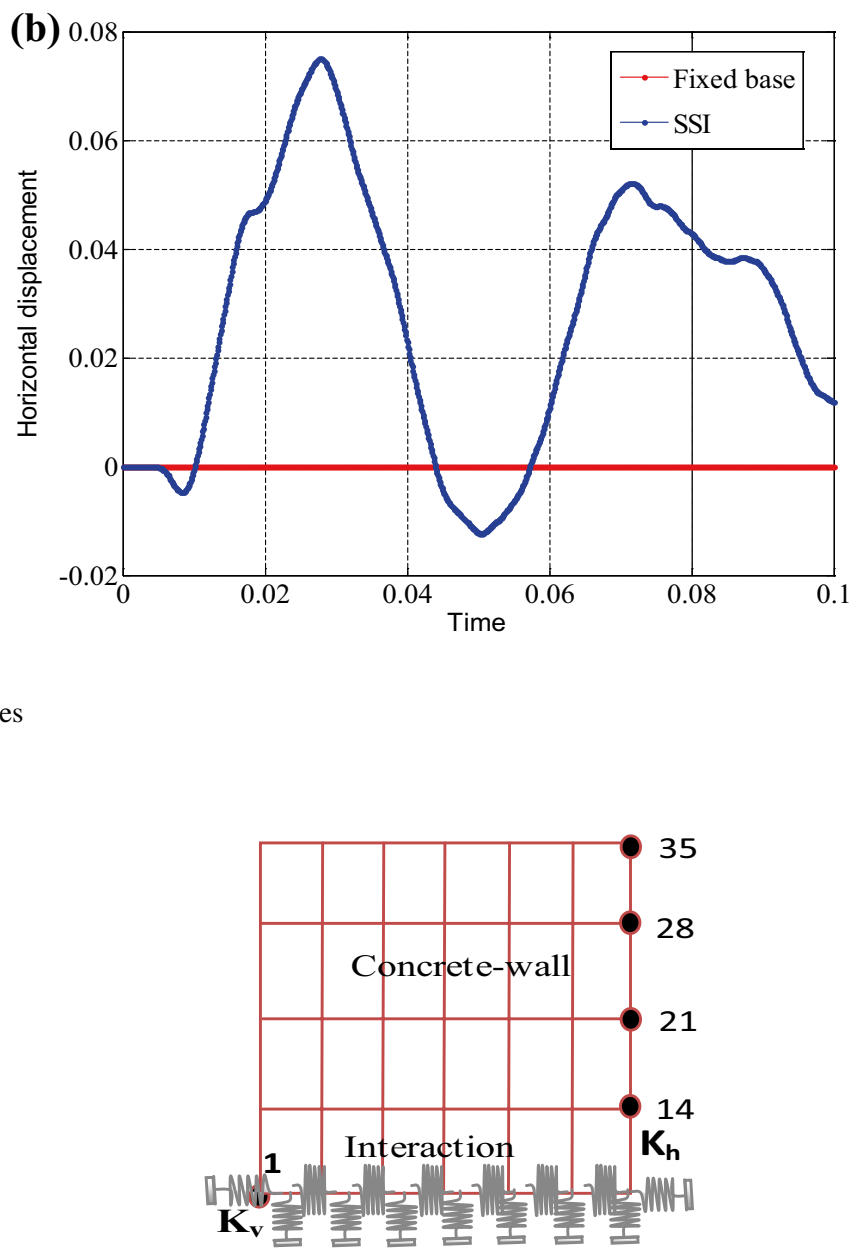

Fig. 9 Model using translational springs

the surrounding and supporting the soil under foundation. Average values of translational springs have been estimated using expressions developed by Newmark and Rosemblueth (1971).

\section{Influence of translational springs}

Figure 10 shows the behavior of the interface medium between the concrete wall and the soil. In this case, the impedance functions used of the soil foundation show an improvement response of $\left(K_{h}+K_{v}\right)$ than those of soil-structure interaction model.

Thus, the impedance functions substituting the soil of foundation can predict highly the dynamic soil-structure interaction. Consequently, spring stiffens taken as average values between stiffness of concrete and the soil predict well the seismic loading. 
(a)

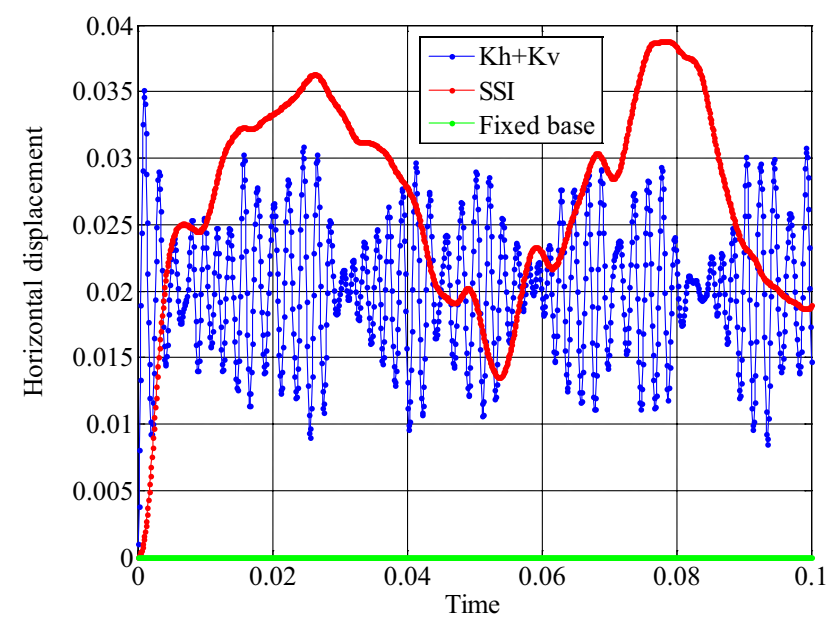

Fig. 10 Influence of spring on $\mathbf{a}$ interface nodes $\mathbf{b}$ concrete wall nodes

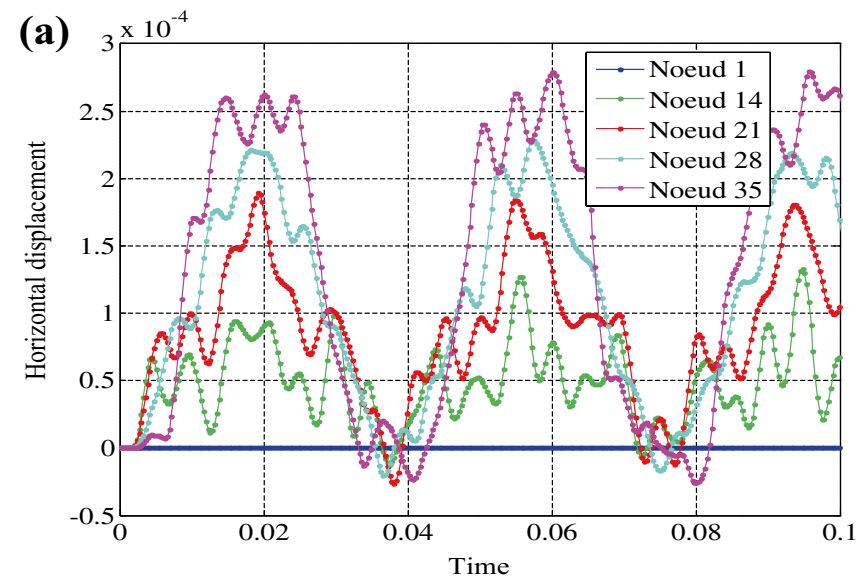

(b)

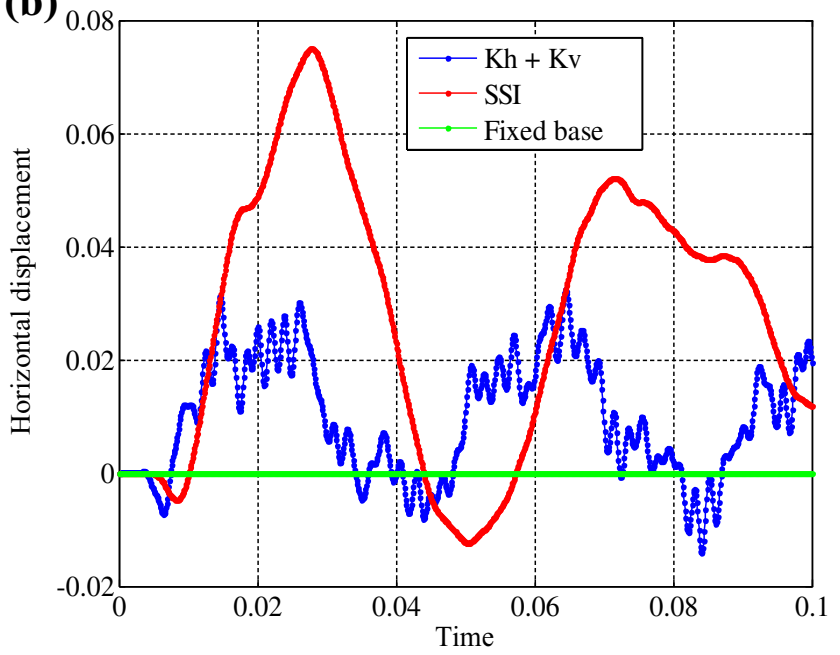

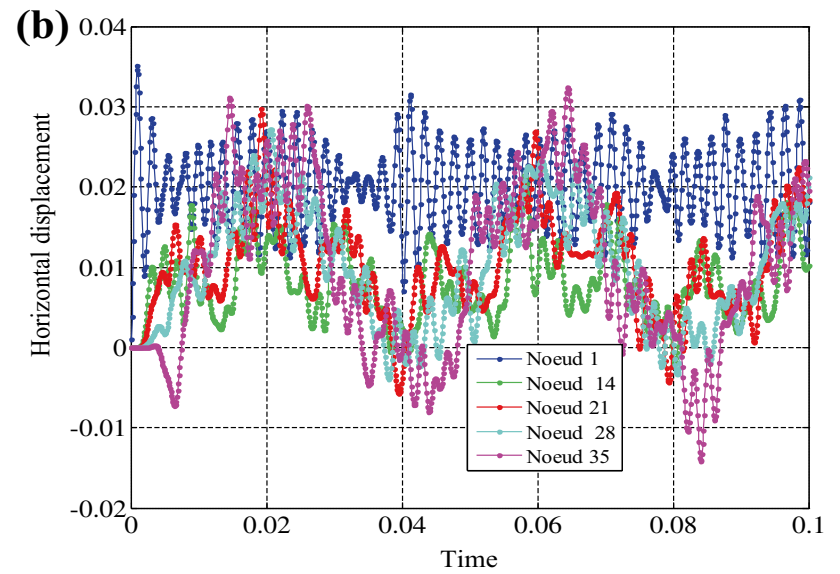

(c)

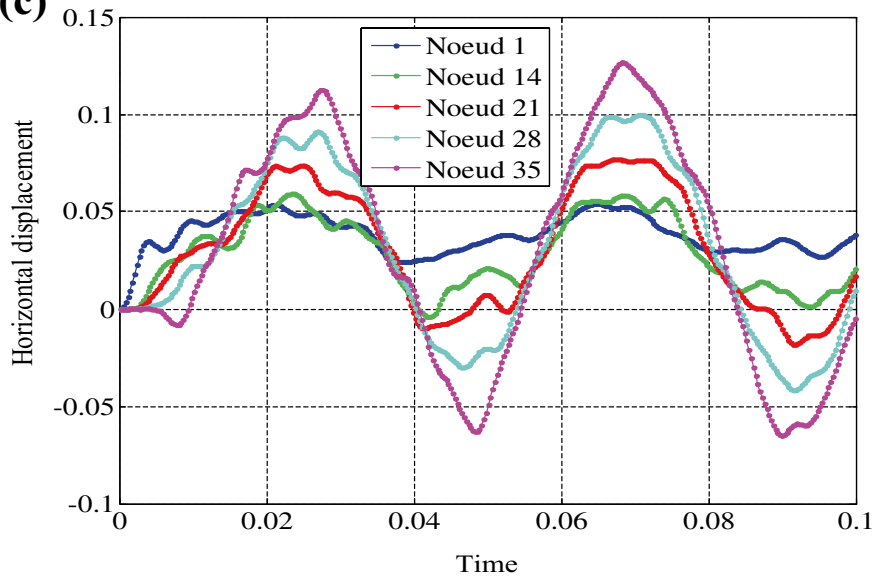

Fig. 11 Horizontal displacements of wall nodes: a fixed-base wall b spring-wall c SSI-wall 


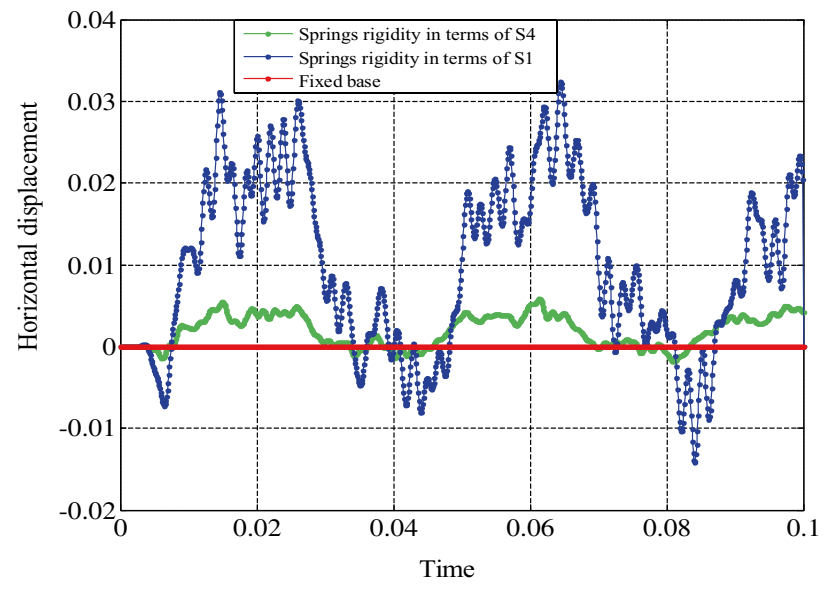

Fig. 12 Stiffness spring effect

\section{Effect of soil-structure interaction on concrete wall}

Our goal is devoted to the analysis of the structural behavior; for this reason, the displacements of the concrete wall nodes are computed. Obtained results (Fig. 11) show horizontal displacements under seismic loading considering (1) fixed-base structure (Fig. 11a), (2) impedance functions (Fig. 11b), and (3) soil-structure interaction model (Fig. 11c). Horizontal displacement values are important for soil-structure interaction and become less with impedance functions and fixed-base structure.

\section{Spring rigidity effect on the concrete wall}

Finally, Fig. 12 shows horizontal displacements of the concrete wall on soft soil (S1), stiff soil (S4), and with fixedbase structure. When the properties of the soil are very well (S4), the structural response in terms of horizontal displacements are considerable compared to those of soft soil. In this case, the behavior of the concrete wall on S4 is improved by $4 \%$ when compared to that on $\mathrm{S} 1$.

\section{Conclusions}

In this paper, the effects of the soil properties and the soil-structure interaction have been studied using nodeto-node contact elements between the supporting soil and the concrete wall. Obtained results using the developed FE program are as follows:

- The flexibility of the soil foundation reduces, notably, the seismic response of the concrete wall and its neglecting leads to larger values of displacements of concrete wall nodes.
- Displacements of concrete wall nodes are important relative to the interaction medium nodes when the foundation is so rocking.

- Damage of the concrete wall base is highly affected by the soil-structure interaction.

- The developed numerical program is an open tool to be improved for non-linear or elastoplastic analyses.

- Formulated analytical model using central difference method of the soil can reproduce considerably the soilstructure interaction.

- Horizontal and vertical behaviors of the interface medium and the wall concrete depend directly on the soil properties. When feeble properties of the soil are used, horizontal and vertical displacements of the concrete wall nodes are largely observed and the horizontal displacements are more pronounced compared to the vertical displacements.

Open Access This article is distributed under the terms of the Creative Commons Attribution 4.0 International License (http://creativeco mmons.org/licenses/by/4.0/), which permits unrestricted use, distribution, and reproduction in any medium, provided you give appropriate credit to the original author(s) and the source, provide a link to the Creative Commons license, and indicate if changes were made.

\section{References}

Barros RC, de Vasconcelos LAC, Nogueira CL, Silveira RAM (2017) Interface elements in geotechnical engineering- some numerical aspects and applications. In: Proceedings of the 38 Latin-American congress on computational methods in engineering, Brazil

Bourouaiah W, Khalfallah S, Guerdouh D (2017) Effect of soil properties on RC wall system responses. Tech J 11(1-2):1-6

Bouzid DA, Tiliouine B, Vermeer PA (2004) Exact formulation of interface stiffness matrix for axi-symmetric bodies under nonaxi-symmetric loading. Comput Geotech 31:75-87

Challamel N, Picandet V, Collet B, Michelits T, Elishakoff I, Wang CM (2015) Revisiting finite difference and finite element methods applied to structural mechanics within enriched continua. Eur J Mech A Solids 53:107-120

Chatzigogos C, Pecker A, Salençon J (2007) A macro-element for dynamic soil-structure interaction analyses of shallow foundations. In: 4 International conference on earthquake geotechnical engineering, Thessaloniki, Greece

Day RA, Potts DM (1994) Zero thickness interface elementsnumerical stability and application. Int J Numer Anal Methods Geomech 18:689-708

Dolicanin CB, Nikolic VB, Dolicanin CD (2010) Application of finite difference method to study of the phenomenon in the theory of thin plates. App Math Inf Mech 2(1):29-43

Fedeliński P (2004) Boundary element method in dynamic analysis of structures with cracks. Eng Anal Bound Elem 28(9):1135-1147

Figini R, Paolucci R, Chatzigogos CT (2012) A macro-element model for non-linear soil-shallow foundation-structure interaction under seismic loads: theoretical development and experimental validation on large scale tests. Earthq Eng Struct Dyn 41(3):475-493 
Gernot B, Ian S, Christian D (2008) The boundary element method with programming: for engineers and scientists. Springer, New York

Ghobarah A (2001) Performance-based design in earthquake engineering: state of development. Eng Struct 23:878-884

Grobeholz G, Delfim SJ, Estorff OV (2015) A stabilized central difference scheme for dynamic analysis. Int J Numer Methods Eng 102(11):1750-1760

Gullu H, Pala M (2014) On the resonance effect by dynamic soilstructure interaction: a revelation study. Nat Hazards 72:827-847

$\mathrm{Hu} \mathrm{L,} \mathrm{Pu} \mathrm{J} \mathrm{(2004)} \mathrm{Testing} \mathrm{and} \mathrm{modeling} \mathrm{of} \mathrm{soil-structure} \mathrm{interface.}$ J Geotech Geoenviron Eng 130(8):851-860

Kwon OS, Elnashai AS (2017) Distributed analysis of interfacing soil and structural systems under dynamic loadings. Innov Infrastruct Solut 2:30

Langen H, Vermeer PA (1991) Interface elements for singular plasticity points. Int J Numer Anal Methods Geomech 15:301-315

Lu Y, Hajirasouliha I, Marshall AM (2016) Performance-based seismic design of flexible-base multi-storey buildings considering soil-structure interaction. Eng Struct 108:90-103

Mahmoudpour S, Attamejad R, Behnia C (2011) Dynamic, analysis of partially embedded structures considering soil structure interaction in time domain. Math Probl Eng. https://doi. org/10.1155/2011/534968

Mayer M, Gaul L (2005) Modeling of contact interfaces using segment-to-segment-elements for FE vibration element. In: Conference and exposition on structural dynamics. Society for Experimental Mechanics

Mekki M, Elachachi SM, Breysse D, Nedjar D, Zoutat M (2014) Soil-structure interaction effects on RC structures within a performance-based earthquake engineering framework. Eur J Environ Civ Eng 18(8):945-962

Mekki M, Elachachi SM, Breysse D, Zoutat M (2016) Seismic behavior of R.C. structures including soil-structure interaction and soil variability effects. Eng Struct 126:15-26
Moghaddasi M, Cubrinovski M, Chase JG, Pampanin S, Carr A (2012) Stochastic quantification of soil-shallow foundation-structure interaction. J Earthq Eng 16:820-850

Newmark NM, Rosemblueth E (1971) Fundamentals of earthquake engineering. Prentice-Hall, Englewood cliffs

Pecker A (2010) Influence of non linear soil structure Interaction on the seismic demand in Bridges, Geodynamics and Structure, Workshop on SSI, Ottawa, 6-8 October 2010

Sharma A (2017) Modelling of contact interfaces using non-homogeneous discrete elements to predict dynamic behaviour of Assembled Structures, Ph.D Technischen Universität Darmstadt

Skejic A (2012) The interface formulation problem in geotechnical finite element software. Electron J Geotech Eng 17:2035-2041

Smith IM, Griffiths DV, Margetts L (2013) Programming the finite element method, programming the finite element method. Wiley, Hoboken, p 682

Viladkar M, Godbole P, Noorzaei J (1994) Modelling of interface for soil-structure interaction studies. Comput Struct 52(4):765-779

Wood WD (2004) Geotechnical modelling. Spoon Press Taylor and Francis Group, London, pp 360-381

Wu B, Deng L, Yang X (2009) Stability of central difference method for dynamic real-time substructure testing. Earthq Eng Struct Dyn 38(14):1649-1663

Zarein F, Krawinkler H (2009) Simplified performance based earthquake engineering, Report $\mathrm{N}^{\circ} 168$, Department of Civil and Environment Engineering. Stanford University, Stanford

Zienkiewicz OC, Taylor RL (2013) The Finite element method for solid and structural mechanics. Elsevier Butterworth-Heinemann, Oxford

Zoutat M, Elachachi SM, Mekki M, Hamane M (2016) Global sensitivity analysis of soil structure interaction system using N2-SSI method. Eur J Environ Civ Eng 22(2):192-211

Publisher's Note Springer Nature remains neutral with regard to jurisdictional claims in published maps and institutional affiliations. 